\title{
Occupational Medicine
}

National Cancer Institute

\section{Source}

National Cancer Institute. Occupational Medicine. NCI Thesaurus. Code C16931.

This branch of medicine is concerned with the prevention, diagnosis, and treatment of injuries and illnesses that occur at the workplace or as a result of toxic exposures, stress, or accidents at the workplace. Occupational medicine encompasses issues such as industrial hygiene, safety, pollution control, ergonomics, industrial and agricultural toxicology, disability evaluation, physical rehabilitation, industrial psychology and emergency medicine. (BioTech Life Science Dictionary) 